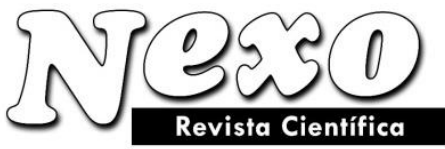

ISSN-E 1995-9516

Universidad Nacional de Ingeniería COPYRIGHT @ (UNI). TODOS LOS DERECHOS RESERVADOS http://revistas.uni.edu.ni/index.php/Nexo https://doi.org/10.5377/nexo.v32i02.9267

\title{
METODOLOGÍA PARA LA RESTAURACIÓN Y PUESTA EN MARCHA DE UNA MÁQUINA FRESADORA CNC
}

\section{METHODOLOGY FOR THE RESTORATION AND COMMISSIONING OF A CNC MILLING MACHINE}

\author{
G. Reyes-Morales ${ }^{1, *}$, V. Guerrero-Hernandez ${ }^{2}$, B.N. Ríos-Ataxca, ${ }^{3}$ O. Ortiz-Aguilar ${ }^{4,}$ E. Cruz- \\ Lázaro $^{1}$ \\ ${ }^{1}$ Tecnológico Nacional de México/Instituto Tecnológico Superior de San Andrés Tuxtla. \\ Ingeniería Mecatrónica. Veracruz, México. \\ ${ }^{2}$ Tecnológico Nacional de México/Instituto Tecnológico Superior de San Andrés Tuxtla. \\ Ingeniería Informática. Veracruz, México. \\ ${ }^{3}$ Tecnológico Nacional de México/Instituto Tecnológico Superior de San Andrés Tuxtla. \\ Ingeniería Electromecánica. Veracruz, México. \\ ${ }^{4}$ Tecnológico Nacional de México/Instituto Tecnológico Superior de San Andrés Tuxtla. \\ Maestría en Ingeniería. Veracruz, México. \\ *greyesm_13@hotmail.com
}

(recibido/received: 14-Mayo-2019; aceptado/accepted: 15-Agosto-2019)

\begin{abstract}
RESUMEN
El Control Numérico Computarizado ( $\mathrm{CNC}$ ) es una tecnología que permite mediante el uso de una computadora controlar y monitorear los movimientos de una máquina herramienta. El Instituto Tecnológico Superior de San Andrés Tuxtla (ITSSAT) cuenta con una máquina herramienta fresadora CNC con tecnología europea adquirida por el gobierno del Estado en el 2003 para prácticas de los estudiantes de ingeniería. La máquina se encontraba obsoleta y sin funcionamiento, situación que provoco no hacer uso de la $\mathrm{CNC}$ en las prácticas durante un tiempo, ya que las industrias que las fabrican utilizan software con licencia y tarjetas electrónicas que tienen un costo elevado para su reparación. El presente artículo tiene como finalidad dar a conocer una metodología para la restauración y puesta en marcha de la máquina fresadora CNC sin necesidad de recurrir al fabricante. Los resultados muestran la metodología cuya principal función es la comunicación de la máquina CNC con la PC mediante la implementación del software alterno Mach 3 y la tarjeta electrónica LPT-Mach 3 logrando el funcionamiento de la máquina y con ello el fresado de materiales con fines académicos e industriales.
\end{abstract}

Palabras claves: Control numérico; Máquina fresadora; Tarjeta electrónica; Automatización; Tecnología $\mathrm{CNC}$

\begin{abstract}
The Computerized Numerical Control (CNC) is a technology that allows, through the use of a computer, to control and monitor the movements of a machine tool. The Superior Technological Institute of San Andrés Tuxtla (ITSSAT) has a CNC milling machine tool with European technology acquired by the state government in 2003 for engineering student practices. The machine was obsolete and out of operation, a situation that obligates not to use the $\mathrm{CNC}$ in practice for a while since the industries that manufacture them use licensed software and electronic cards that have a high cost for repair. The purpose of this article is to
\end{abstract}

Nexo Revista Científica / Vol. 32, No. 02, pp. 179-189 / Diciembre 2019 


\section{G. Reyes-Morales et al.}

present a methodology for the restoration and start-up of CNC machines without having to resort to the manufacturer. The results show the methodology whose main function is the communication of the CNC machine with the PC through the implementation of the alternate software Mach 3 and the electronic card LPT-Mach 3 achieving the operation of the machine and the milling of materials with academic and industrial purposes.

Keywords: Numerical control; Milling machine; Electronic card; Automation; CNC technology

\section{INTRODUCCIÓN}

El Control Numérico Computarizado $(\mathrm{CNC})$ es una tecnología que permite mediante el uso de una computadora controlar y monitorear los movimientos de una máquina herramienta (Amor, 2018). Los antecedentes nos muestran que la tecnología de control numérico $(\mathrm{CN})$ dio inicio en 1945 con los científicos John Manclhyn y J. Prester, creadores de la primer súper computadora ENIAC, fue el punto de partida para el control numérico, pero realmente hasta 1948 llego la aplicación del control numérico a una máquina herramienta, John Person dio solución a la problemática del fresado de superficies complejas tridimensionales en la industria de la aeronáutica (Castro y Martínez).

En 1962 George Amber y Paul Amber propusieron una escala patrón de la automatización fundamentada en la idea de que para llevar cualquier trabajo es necesaria la energía humana o energía de una máquina, así como, la información. Derivado de los grandes costos fue hasta en los años 70 cuando surgió el desarrollo de la microelectrónica, dando inicio al CNC.

De acuerdo a la escala patrón la categoría A(5), en este nivel ya es indispensable disponer de un ordenador, el cual se programa con ecuaciones matemáticas que definen el comportamiento del proceso, sus condicionantes y sus aspectos que deben ser optimizados (Amor, 2018). Por lo tanto, las fresadoras CNC son una forma de automatización programable en la que el proceso se controla mediante un sistema alfanumérico de números, letras y símbolos.

Una máquina $\mathrm{CNC}$ ofrecen alta competitividad en las industrias con respecto al manufacturado de piezas del tipo 2D \& 3D, pues son empleadas como una tecnología clave en el campo de la fabricación de manufactura avanzada (Xiong-Bo, M.A. et al., 2007). En la figura 1 se muestran las partes principales implicadas en un sistema de manufacturado CNC.

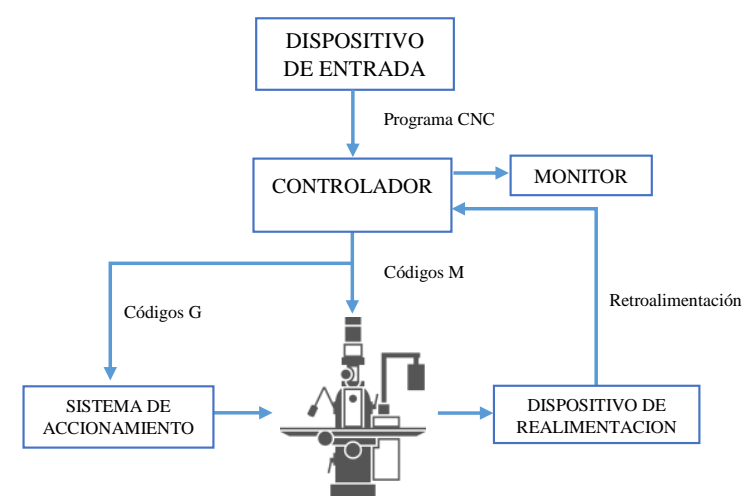

Figura 1. Diagrama de Bloques de una Máquina Fuente: (Elaboración propia)

Para realizar el control de movimiento de una maquinaria CNC es necesario procesar los datos a través del controlador y de un sistema de control computarizado, dicho sistema es considerado a menudo como el 


\section{G. Reyes-Morales et al.}

software de control de la máquina (Osornio-Rios, R. et al, 2008). El software de control por defecto es proporcionado al adquirir una maquinaria $\mathrm{CNC}$, y así mismo es adjuntado con una clave de dicho producto, tal software puede ser descargado vía internet desde la página oficial de la empresa, sin embargo, la clave del producto no se proporciona de forma gratuita, y de ser extraviada o dañada dicha clave tiene que comprarse, siendo los costos alrededor de $£ 484.50$ a $£ 1445$ o su equivalente $\$ 11931.88$ MN a $\$ 35586.30$ $\mathrm{MN}$, siendo esto una inversión económicamente alta.

Recientemente en el mercado industrial, han surgido nuevos software de control como alternativas a los sistema de control computarizado que vienen por defecto en las maquinarias CNC (Yusof, Y., Latif, K. 2013), dicho software funcionan como una interfaz que permite la simplificación del proceso de programación y manufacturado de piezas 2D \& 3D. Dependiendo de las necesidades y del uso del software que se aplicara, y esto es debido a que también implican un costo medianamente alto, pero menor a los costos del software original de la máquina. Uno de los softwares de control más empleado en el mercado es Mach3 (Bourassa, V. y Zahorhjan, J., 1995) y esto es debido a que permite la configuración entre la interfaz y el controlador de una maquinaria $\mathrm{CNC}$, además de contar con una licencia con un costo aproximado de $\$ 200.00$.

\section{METODOLOGÍA PROPUESTA}

En la figura 2 se muestra el diagrama de la metodología propuesta para la implementación de la puesta en marcha de una Fresadora CNC Denford mediante la aplicación del sistema de control computarizado Mach3. La metodología está basada en emplear los criterios bases (Newman, S. T., Allen, R. D. y Rosso, J., 2003) para que dichos métodos a mencionar puedan ser aplicados a otras maquinarias CNC, como lo son el caso de Tornos CNC y Centros de Maquinados Verticales (VMC), explicando la identificación de los componentes electrónicos principales implicados en el controlador de la máquina basados en el estándar (Latif, K., Yusof, Y., 2016), para poder ser conectados con una interfaz de control capaz de comunicarse con Mach3. Con ello se propone un sistema integrado para la automatización de maquinarias CNC, los cuales permitirían desarrollar a un bajo costo la actualización de equipos CNC en instituciones educativas o poder ser aplicados en pequeñas y medianas industrias (Xu, X. W. et al, 2005; Milojkovic, M. T. et al, 2014).

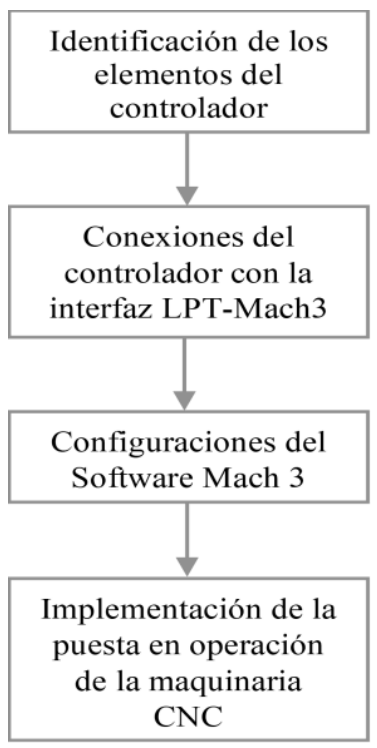

Figura 2. Metodología propuesta Fuente: (Elaboración propia).

Nexo Revista Científica / Vol. 32, No. 02, pp. 179-189 / Diciembre 2019 


\section{G. Reyes-Morales et al.}

A continuación, se explican cada uno de los pasos de la metodología aplicada para la puesta en marcha de la máquina $\mathrm{CNC}$ :

\subsection{Identificación de los elementos del controlador}

En esta etapa se analizan dos aspectos principales; el primero es el elemento mecánico, los cuales corresponden a los tipos de motores que se emplean para dar movimiento a los ejes X, Y y Z, límites de carreras, así como el tipo de motor de husillo que emplea la maquinaria CNC, el segundo son los elementos de control, en esta etapa se involucran aspectos ligados a la electrónica digital y de potencia, sensores, drivers, acondicionamiento y control de señales.

\subsection{Conexiones del controlador con la interfaz LPT-Mach3:}

Mach3 es una interfaz que se comunica directamente a través del puerto paralelo, por lo que la interacción se realiza a través de sus pines; para realizar la comunicación correcta se emplea una interfaz DB25 de pines electrónicos mostrada en la figura 3.

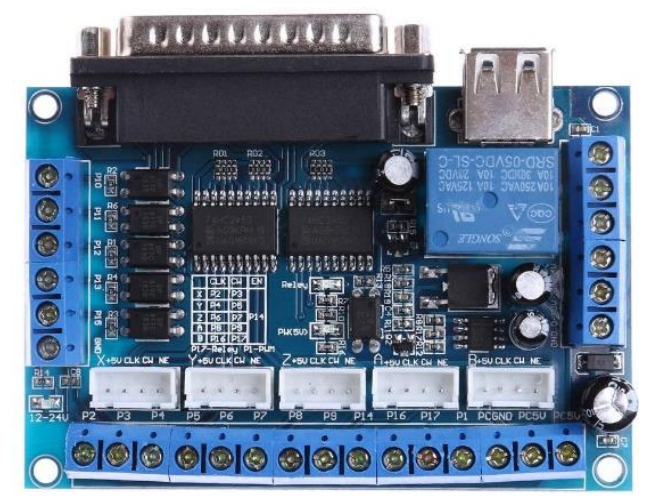

Figura 3. Tarjeta de control DB25

Fuente: (Manual LPT-Mach3).

Dicha tarjeta cuenta con pines básicos para el control de una maquinaria $\mathrm{CNC}$, en las cuales sus funciones son:

- $\quad$ Soporte para 5 Ejes

- $\quad$ Soporte para límites de ejes X, Y \& Z

- Soporte para Botón de Paro de Emergencia

- Soporte para Zonda de Cero automático

- Salida PWM de 0-10 VDC

Permitiendo que con dichas opciones se realice el control de una maquinaria CNC con sus funciones básicas. Para conectar dicha placa $D B 25$ con el controlador de la máquina CNC, se analizó el sistema principal de control mostrada en la figura 4, dicha placa pertenece al modelo Smart Step3, que cuenta con un conector del tipo DIN de 96 pines, dicho conector incluye entradas de interruptores de límite, salidas con opciones de amplificadores y salidas de error, la ubicación de este conector se muestra en la figura 4 marcada mediante un recuadro rojo.

Nexo Revista Científica / Vol. 32, No. 02, pp. 179-189 / Diciembre 2019 


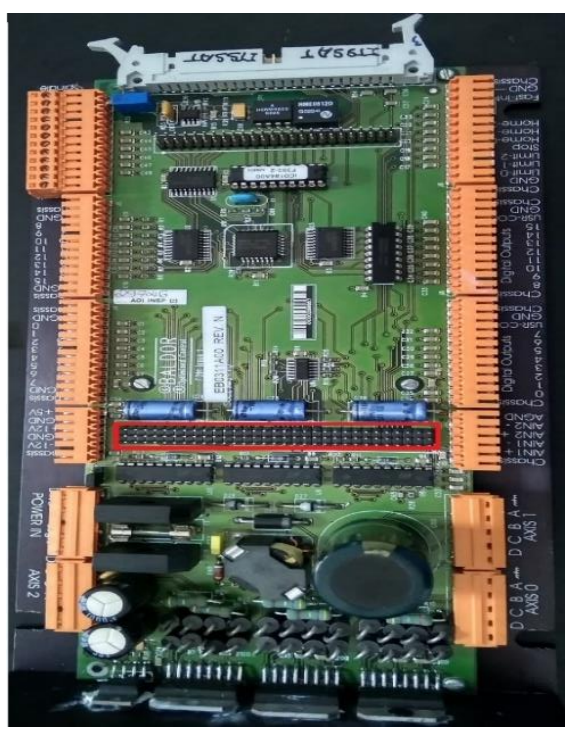

Figura 4. Tarjeta de control de la máquina CNC Fuente: (Elaboración propia).

\subsection{Configuraciones del Software Mach 3}

En la tabla 1 se muestra los 96 pines de conexión de la tarjeta controladora CNC Smart Step 3 de la máquina CNC. Con dichos datos se realizan las conexiones correspondientes entre la interfaz DB25 y el controlador Smart Step3, mostrándose en la figura 5.

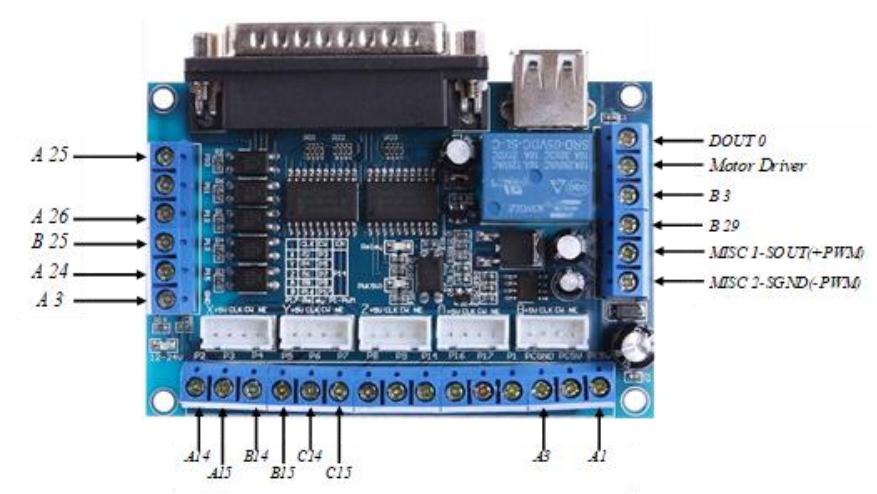

Figura 5. Conexión de la interfaz DB25 Fuente: (Manual LPT-Mach3). 
Tabla 1. Conector de 96 pines in-out

\begin{tabular}{|c|c|c|c|}
\hline No. & $\mathrm{C}$ & B & A \\
\hline 1 & $+5 \mathrm{~V}$ & $+5 \mathrm{~V}$ & $+5 \mathrm{~V}$ \\
\hline 2 & $+5 \mathrm{~V}$ & $+5 \mathrm{~V}$ & $+5 \mathrm{~V}$ \\
\hline 3 & GND & GND & GND \\
\hline 4 & USR-OUT-6 & USR-OUT-7 & USR-OUT-COM \\
\hline 5 & USR-OUT-3 & USR-OUT-4 & USR-OUT-5 \\
\hline 6 & USR-OUT-0 & USR-OUT-1 & USR-OUT-2 \\
\hline 7 & CHB-1 & CHA-0 & CHB-0 \\
\hline 8 & IDX-1 & IDX-0 & CHA-1 \\
\hline 9 & !CHA-1 & !!IDX-1 & !IDX-1 \\
\hline 10 & !CHB-0 & !CHA-0 & ¡ICHB-1 \\
\hline 11 & ERROR-IN & ERROR-OUT & GND \\
\hline 12 & RESET-IN & GND & GND \\
\hline 13 & GND & BOOST-1 & BOOST-0 \\
\hline 14 & PULSE-2 & PULSE-1 & PULSE-0 \\
\hline 15 & DIR-2 & DIR-1 & DIR-0 \\
\hline 16 & BOOST-2 & GND & DSR \\
\hline 17 & GND & GND & DTS \\
\hline 18 & USR-IN-4 & FAST-IN & USR-IN-2 \\
\hline 19 & USR-IN-3 & USR-IN-5 & USR-IN7 \\
\hline 20 & USR-IN-6 & USR-IN-1 & RXD \\
\hline 21 & USR-IN-0 & RTS & TXD \\
\hline 22 & OPT-4 & OPT-2 & CTS \\
\hline 23 & H0ME-2 & OPT-3 & OPT1 \\
\hline 24 & PULSE-IN & HOME-1 & LIMIT-2 \\
\hline 25 & DIR-IN & LIMIT-1 & STOP \\
\hline 26 & HOME-0 & RESET-CNTR & LIMIT-0 \\
\hline 27 & DEMAND-0 & DEMAND- & -ANALOGUE-2 \\
\hline 28 & +ANALOGUE-2 & +ANALOGUE-1 & -ANALOGUE-1 \\
\hline 29 & $+12 \mathrm{~V}$ & $+12 \mathrm{~V}$ & $+12 \mathrm{~V}$ \\
\hline 30 & AGND & AGND & AGND \\
\hline 31 & $-12 \mathrm{~V}$ & $-12 \mathrm{~V}$ & $-12 \mathrm{~V}$ \\
\hline 32 & SCRN & SCRN & SCRN \\
\hline
\end{tabular}

Para el control del movimiento de los ejes es necesario de la implementación del software para el manejo de las señales de salida por el puerto paralelo, Mach3 cuenta con una gran versatilidad para aplicaciones CNC (Newman, S. T., Allen, R. D. y Rosso, J., 2003), la pantalla principal de sistema se muestra en la figura 6.

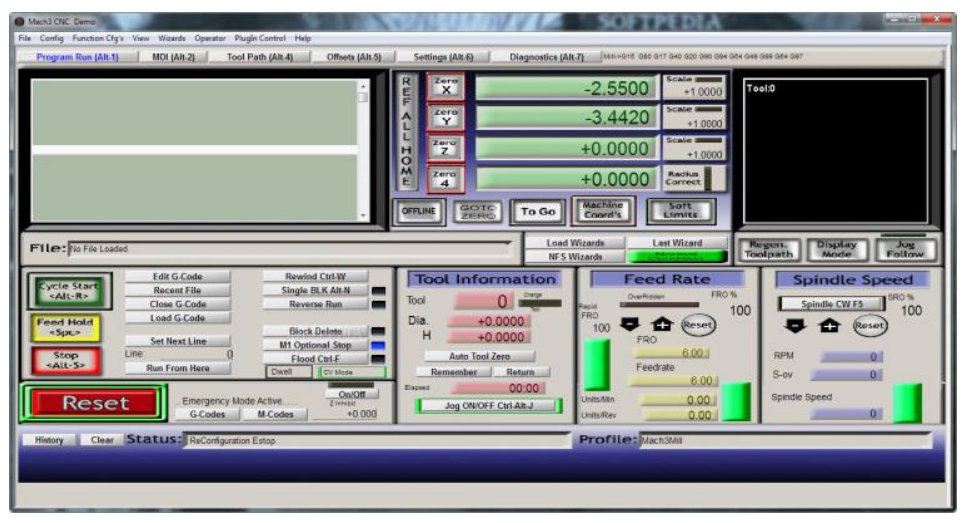

Figura 6. Pantalla principal de Mach3 Fuente: (Software Mach3). 


\section{G. Reyes-Morales et al.}

Para la configuración de la aplicación, es necesario considerar dos secciones principales, Ports and Pins. En la opción de Port Address se mantiene por defecto 0x378, en la opción de Kernel Speed se debe de escoger por defecto $25000 \mathrm{~Hz}$, con dicha velocidad de la transmisión de datos es más que satisfactoria, de aumentar la velocidad los datos serán leídos en menor tiempo, pero con la probabilidad de perdida de datos. En el apartado de Motor Outputs se designan los pines para los respectivos motores de los ejes $X, Y \& Z$, siendo dichas configuraciones las mostradas en la tabla 2.

Tabla 2. Configuración de pines Mach3

\begin{tabular}{ccc}
\hline & Pin Smart Step & Pin Mach 3 \\
\hline \multirow{2}{*}{ Eje X } & Pulse-0 & 2 \\
& Dir-0 & 3 \\
& Limit -0 & 12 \\
Eje Y & Pulse-1 & 4 \\
& Dir-2 & 5 \\
& Limit -1 & 13 \\
Eje Z & Pulse-2 & 6 \\
& Dir-2 & 7 \\
Botón de Paro de & Limit -2 & 15 \\
Emergencia & Stop & 10 \\
Spindle & DOUT-0 & 17 \\
\hline
\end{tabular}

Fuente: (Manual LPT- Mach3).

Adicionalmente, para activar la opción de $P W M$ y con ello controlar la velocidad del Husillo, en el apartado de Outputs Signals se debe de activar la casilla de Output \#1 con la configuración de Port\#=1 y Pin Number=1. Y posterior a ello, en el apartado de Spindle Setup, activar la casilla de Use Spindle Setup y PWM Control.

Finalmente, el Motor Tunnig se debe de configurar el movimiento sobre los motores de los respectivos ejes, con dichas opciones se configuran la cantidad de pulsos a enviar, la velocidad de respuesta del motor, así como aceleración y desaceleración, tal como se muestra en la figura 7.

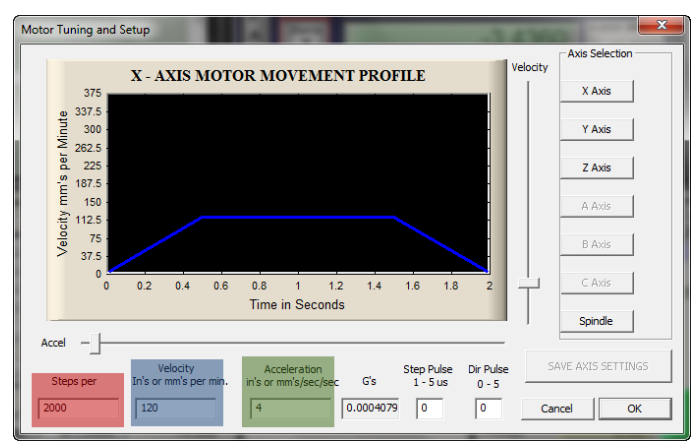

Figura 7 Configuración de los Ejes (Motores) Fuente: (Software Mach3).

\subsection{Implementación de la puesta en operación de la máquina $C N C$.}

Con las configuraciones correctas, así como la calibración adecuada (Romero-Troncoso R. et al, 2003), la puesta en marcha de la máquina CNC puede ser implementado mediante el diseño de piezas del tipo 2D \& 3D. 


\section{G. Reyes-Morales et al.}

\section{RESULTADOS.}

La validación de la metodología de restauración de la máquina herramienta CNC se llevó a cabo mediante un caso de estudio de maquinado en madera, en las siguientes secciones se describen los resultados.

\subsection{Diseño $C A D$}

Para este caso de estudio, se diseñaron diferentes modelos 2D mediante el uso de un Software de diseño CAD, el software de diseño empleado fué Inkscape V0.92, proporcionando herramientas de dibujo adecuadas para el diseño de arcos y círculos, los cuales con dichas representaciones vectoriales hacen que la máquina muestre (de existir el caso) posibles errores en los movimientos de los ejes, permitiéndonos guardar el diseño en un formato del tipo DXF compatible con la mayoría de software CAM compatible con el modelo (Raksiri, C., Parnichkun, M., 2004). Para este caso se diseñó un logo de la carrera de ingeniería Mecatrónica, mostrado en la figura 8.

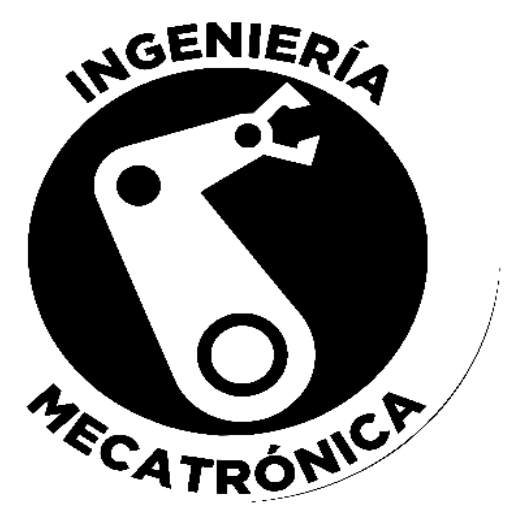

Figura 8. Diseño de figura CAD. Fuente: (Software Inkscape V0.92).

\subsection{Procesamiento CAM}

Realizado el diseño CAD de la figura que se va a maquinar como se mostró en el apartado 3.1, se continua con la simulación del software como se muestra en la figura 9 (a), y se permanece con el procesamiento CAM del diseño realizado, se emplea el software MasterCAM 2017, se muestra el diseño de la pieza bajo el proceso de simulación mostrando los resultados en la figura 9 (b).

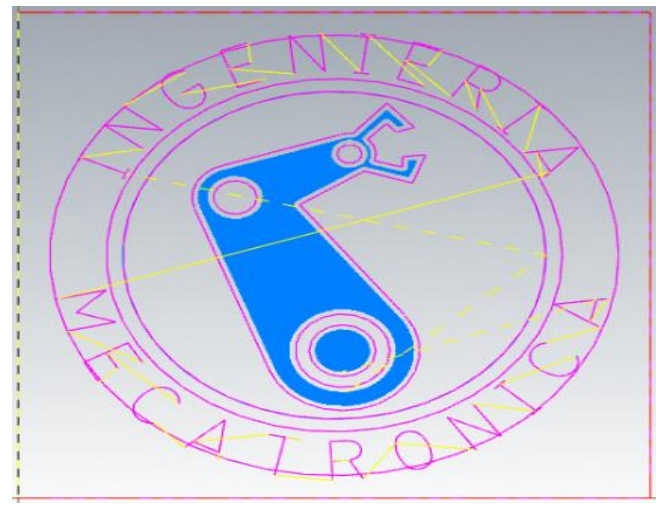

(a)

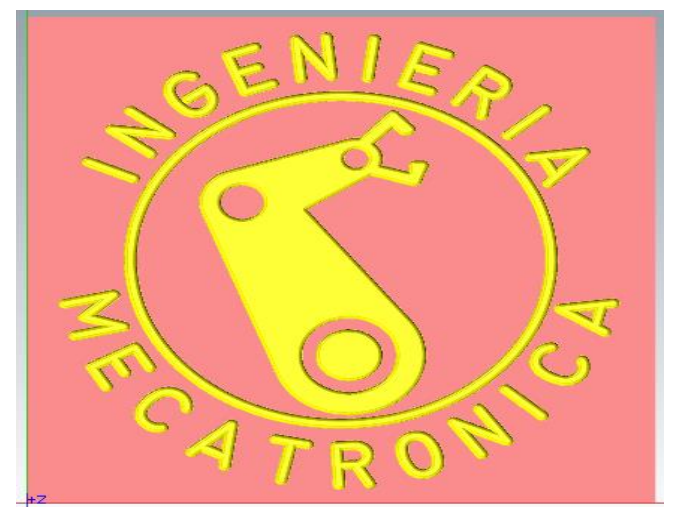

(b)

Figura 9. Simulación de la pieza a maquinar. (a) Pieza Diseñada en CAD. (b) Pieza simulada en CAD Fuente: (Software MasterCAM, 2017). 


\subsection{Manufacturado $2 D$}

Durante el manufacturado de dicho diseño, se obtuvieron ligeras diferencias de medidas con respecto al diseño $y$ al resultado final del Manufacturado 2D, tales diferencias tenían un margen de error de $\pm 1 \mathrm{~mm}$. Siendo dicha diferencia un margen mínimo, para corregirlo, Mach3 cuenta con una opción de calibración de los ejes llamada SET STEPS PER UNIT. Dicha opción se encuentra en la pantalla principal de Mach3 en la opción de SETTINGS ALT6 mostrada en la figura 10.

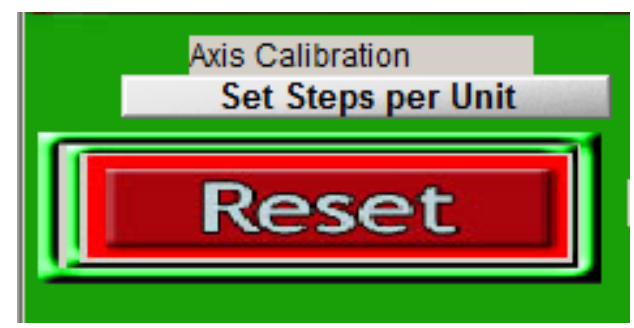

Figura 10. Calibración de Ejes en Mach3

Fuente: (Software Mach3).

En dicha opción de calibración de ejes, se le indicó al programa la posición al cual se moverá el eje, posterior a ello cuando la máquina se posiciono en la coordenada indicada, se analizó mediante un Calibrador vernier si la posición indicada correspondía a la correcta, al existir mínimas diferencias se ingresó la posición obtenida con el Calibrador y de manera automática Mach3 muestra una sugerencia de calibración del eje a calibrar. Con dichas acciones y repitiendo el procedimiento para cada uno de los ejes se mejora el acabado superficial (Baptista, R., Simoes, JF A., 2000), se realizó nuevamente la manufactura del diseño realizado, obteniendo resultados muy satisfactorios mostrados en las figuras 11(a), 11(b) y 11(c).

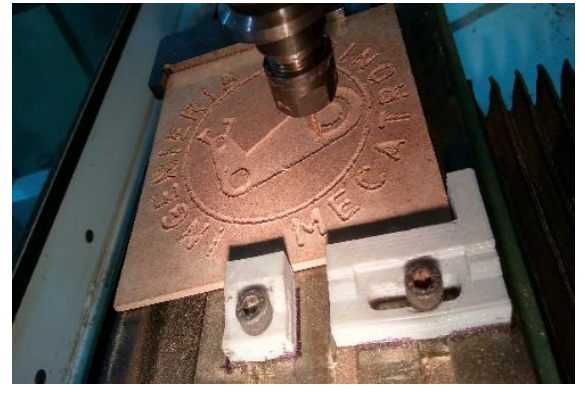

(a)

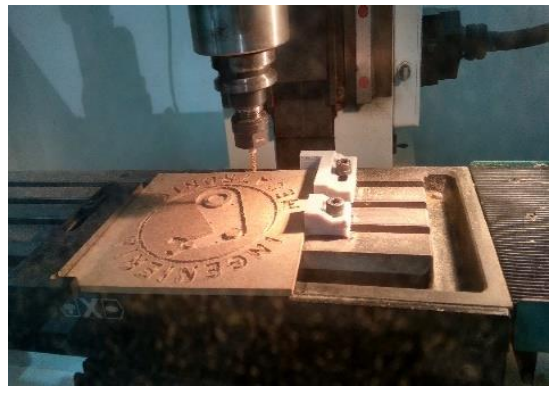

(b)

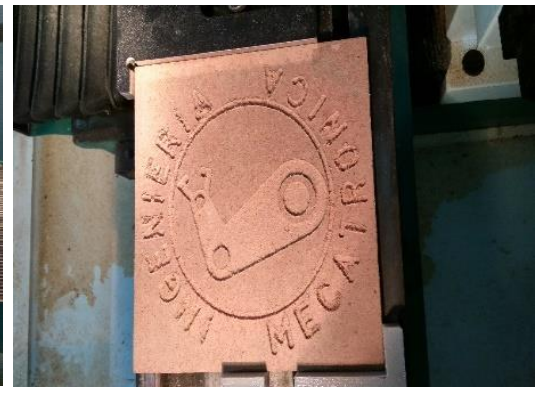

(c)

Figura 11. Logo de Mecatrónica maquinado en madera. (a) Inicio del maquinado en la CNC.

(b) Terminación del maquinado en la CNC. (c) Resultado final del maquinado. Fuente: (Elaboración propia).

\section{CONCLUSIONES}

Mediante la metodología presentada, se demostraron los pasos elementales para la configuración del Software Mach3, restaurar y poner en marcha la fresadora máquina herramienta CNC. El logro de dicha interacción, se partió de un análisis a fondo de los componentes del controlador de dicha maquinaria, esto con el fin de sustituir el software de control que realiza la interacción con la máquina y el movimiento de los ejes. Así mismo, mediante la adaptación del software, es posible usar cualquier otro software de procesamiento $C A M$ teniendo la seguridad de poder leer los códigos $G \& M$ generado por dicho software, además de poder implementar mejoras o actualizaciones a la máquina, y mediante la realización de Macros en Mach 3 es posible poder controlar entradas y salidas externas. Con la implementación de dicho sistema 


\section{G. Reyes-Morales et al.}

de control se demuestra una alternativa y solución a las maquinarias CNC que no cuentan con un software de control, y con la aplicación de esta, se reduce el costo de inversión. Los resultados presentados con la placa de interfaz DB25 que solo cuenta con pines elementales, fue demostrar el control de los motores de paso de la mááquina $\mathrm{CNC}$ para la puesta en marcha, que es el objetivo de esta investigación, y por lo tanto las conexiones y configuraciones realizadas en el software Mach3 se mantienen igual, bastando con realizar un Macro que cumpla con las necesidades de la máquina sobre la que se aplique la implementación. Además de que se pueden realizar las actualizaciones y con esto la maquina CNC deja de ser obsoleta.

\section{REFERENCIAS}

Amor, G. (2018). Evolución, Análisis y Estudio comparativo de las máquinas herramienta (Torno y Fresadora) a lo largo de la historia (Tesis de Ingeniería). Recuperado de http://uvadoc.uva.es/handle/10324/31259.

Baptista, R., Simoes, JF A. (2000). Three and five axes milling of sculptured surfaces. Journal of materials processing technology, 103 (3), 398-403.

Bourassa, V. y Zahorhjan, J. (1995). Implementing lightweight remote procedure calls in the Mach 3 operating system. Department of Computer Science and Engineering, University of Washington. Recuperado de ftp://ftp.cs.washington.edu/tr/1995/02/UW-CSE-95-02-01.pdf

Castro, J. A. y Martínez, J. Máquina compacta usando tecnología CNC para impression 3D y manufactura. Universidad Tecnológica de Tijuana. Ingeniería en Mecatrónica.

Elias, D. M., Yusof, Y., Minhat, M (2013). CNC machine system via STEP-NC data model and LabVIEW platform for milling operation. En 2013 IEEE conference on open systems (ICOS). IEEE. 27-31.

Latif, K., Yusof, Y. (2016). New method for the development of sustainable STEP-compliant open CNC system. Procedia CIRP, 40, 230-235.

Loepere, K. (1991). Mach 3 Kernel Principles (Manual software Mach 3). Recuperado de http://www.shakthimaan.com/downloads/hurd/kernel_principles.pdf

Milojkovic, M. T., Milovanovic, M. B., Mitic, D.V., Peric, S. L., Spasic, M. D. y Nikolie, S. S. (2014). Laboratory CNC machine for education of students in control systems engineering. Facta Universitatis, Series: Automatic Control and Robotics, 13(2), 117-125.

Newman, S. T., Allen, R. D. y Rosso, J. (2003). CAD/CAM solutions for STEP-compliant CNC manufacture. International Journal of Computer Integrated Manufacturing, 16(7-8), 590-597.

Osornio-Rios, R., Romero-Troncoso, R. J., Herrera-Ruiz, G. y Castañeda-Miranda, R. (2008). The application of reconfigurable logic to high speed $\mathrm{CNC}$ milling machines controllers. Control Engineering Practice, 16(6), 674-684.

Raksiri, C., Parnichkun, M., (2004). Geometric and force errors compensation in a 3-axis CNC milling machine. International Journal of Machine Tools and Manufacture, 44, 1283-1291.

Romero-Troncoso R., Herrera-Ruiz, G., Terol-Villalobos, I. y Jauregui-Correa, J. C. (2003). Driver current analysis for sensorless tool breakage monitoring of CNC milling machines. International Journal of Machine Tools and Manufacture, 43(15), 1529-1534.

Nexo Revista Científica / Vol. 32, No. 02, pp. 179-189 / Diciembre 2019 


\section{G. Reyes-Morales et al.}

Xiong-Bo, M.A., Zhen-Yu. H., Yong-Zhang, W., y Hong-Ya, F. (2007). Development of a PC-based open architecture software-CNC system. Chinese Journal of Aeronautics, 20(3), 272-281.

Xu, X. W., Wang, H., Mao, J., Newman, S. T., Kramer, T.R., Proctor, F. M. y Michaloski, J. L. (2005). STEP-compliant NC research: the search for intelligent CAD/CAPP/CAM/CNC integration. International Journal of Production Research, 43(17), 3703-3743.

Yusof, Y., Latif, K. (2013). Frame work of LV-UTHM: AN ISO 14649 Based open control system for CNC milling machine. En Applied Mechanics and Materials. Trans Tech Publications. 330, 619-623.

\section{SEMBLANZA DE LOS AUTORES}

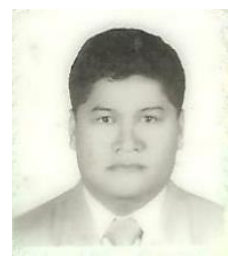

Guillermo Reyes Morales: Obtuvo el grado de Ingeniero Mecánico Electricista por la Universidad Veracruzana, Veracruz, México. Desarrolló sus estudios de Maestría en Ciencias en Ingeniería Electrónica, por el Instituto Tecnológico de Orizaba, Veracruz, México. Ha desarrollado estancia de investigación en la universidad Estatal de Morehead en Estados Unidos de América dentro de su campo de especialidad. Trabaja en líneas de investigación vinculadas con la automatización y control, así como el Diseño y Análisis en la realidad virtual para mejorar el proceso capacitación tecnológica en ingenieros de diferentes especialidades a nivel industrial, actualmente labora en el Instituto Tecnológico Superior de San Andrés, como docente de tiempo completo en el área de Ingeniería Mecatrónica.

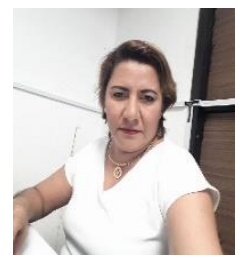

Verónica Guerrero-Hernández: Maestría en Tecnologías de Información por la Universidad Cristóbal Colon de Veracruz, Ver. México y Licenciada en Informática por el Instituto Tecnológico de Orizaba, Veracruz, México. Profesora de tiempo completo en el Instituto Tecnológico Superior de San Andrés Tuxtla (ITSSAT) desde 1997. Reconocimiento de Perfil Deseable PRODEP del 2013 - 2016. Trabaja en líneas de investigación vinculadas con el desarrollo de tecnologías emergentes. Los proyectos de investigación en los que trabaja son sobre entornos virtuales de aprendizaje y la competencia investigadora. Actualmente coordina el Programa Institucional de Semilleros de Investigación (PISI) en el ITSSAT

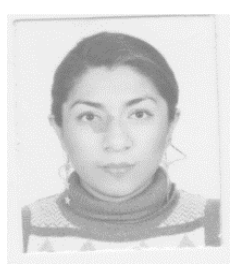

Blanca Nicandria Ríos Ataxca: Obtuvo el grado de Licenciada en Instrumentación Electrónica en la Universidad Veracruzana, Veracruz, México. Desarrolló sus estudios de Maestría en Ingeniería Industrial, por la Universidad Cristóbal Colon, Veracruz, México. Trabaja en líneas de investigación vinculadas con la automatización y control, actualmente labora en el Instituto Tecnológico Superior de San Andrés, como docente de tiempo completo en el área de Ingeniería Electromecánica, Reconocimiento de Perfil Deseable PRODEP 2019 - 2022.

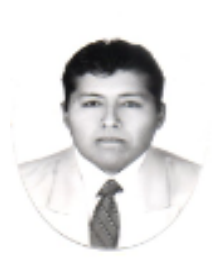

Oscar Ortiz Aguilar: Estudiante de la Maestría en Ingenieras, en el Instituto Tecnológico Superior de San Andrés Tuxtla, Veracruz, México. Obtuvo el grado de Ingeniero Electromecánico en el Instituto Tecnológico Superior de San Andrés Tuxtla, Veracruz, México. Docente en la especialidad de mantenimiento automotriz y colaborador en el área físico-matemáticas de nivel medio superior en Centro de Estudios Tecnológicos Industrial y de servicios No. 110 "José Mariano Michelena". Trabaja en la línea de investigación de automatización industrial y tiene 28 años de experiencia en mecánica automotriz.

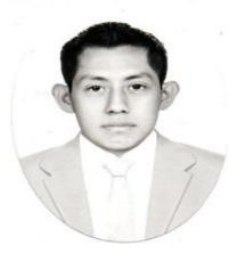

Edi Cruz Lázaro: Alumno de Ingeniería Mecatrónica, por el Instituto Tecnológico Superior de San Andrés Tuxtla, Veracruz, México. Trabaja en líneas de investigación vinculadas con el control y la automatización en desarrollo industrial

Nexo Revista Científica / Vol. 32, No. 02, pp. 179-189 / Diciembre 2019 